\title{
Yellow fever: readily prevented but difficult to treat
}

Background and epidemiology: Yellow fever is a viral hemmorhagic fever endemic in jungle areas and, less commonly, urban areas of South America and Africa. It is caused by a flavivirus that is passed to humans inadvertently through the bite of the Aedes aegypti mosquitoes (urban cycle) or of Haemagogus or other forest-canopy species of mosquitoes (jungle or forest cycle). Endemicity is maintained by the presence of the vector (mosquitoes) and nonhuman primates (monkeys) as the zoonotic focus. ${ }^{1}$

According to estimates by the World Health Organization (WHO) in 1998, each year about 200000 people become ill with yellow fever worldwide, but the true incidence is likely much higher. About $90 \%$ of cases occur in Africa and 10\% in South America. In South America this disease manifests primarily in nonhuman primates, with the peak transmission between January and March, particularly in areas cleared of trees for habitation and agriculture. South America has been epidemic-free since 1942 because of a successful $A$. aegypti eradication program, but the threat of resurgence remains. In Africa, transmission occurs in the tropical rain forest, moist savannah and contiguous dry savannah areas. Peak transmission occurs in the rainy season and early dry season from July through October. Between 1979 and 2002 there were 10 reported cases of imported yellow fever in returning travellers, reflecting increasing global travel to endemic areas. ${ }^{2}$

The incubation period for yellow fever is generally 3-6 $\checkmark$ days. Classic illness passes through 3 defined phases: infection, remission and intoxication. Not all cases progress through the 3 phases. Mild illness characterized by fever, prostration, headache, myalgia, abdominal pain and vomiting may resolve immediately and permanently following the period of infection. More severe cases may progress through a period of remission, marked by a sudden fall in fever lasting several hours or days, to intoxication. The period of intoxication presents with jaundice, azotemia, oliguria, myocardial, renal and hepatic involvement, encephalopathy and hemorrhage, characterized by hematemesis. People with yellow fever have a $10 \%$ mortality rate; this rate may climb as high as $50 \%$ in cases of hemorrhagic fever.

People of all ages are equally susceptible, but in epidemics those who are too young to have been immunized during previous epidemics are more vulnerable. A large proportion of cases worldwide are in men aged 15-45, reflecting occupational exposure through forestry or agricultural work in mosquitoridden jungle areas. Unlike other hemorrhagic fevers, yellow fever is not directly transmitted from person to person. Impoverished areas with poor health care, limited access to immunization and limited re-

sources for reducing exposure to mosquitoes have the highest rates of cases.

Clinical management: Three questions on patient history that could suggest yellow fever are outlined in Table 1.3 Signs during the infection phase include fever, Faget's sign (bradycardia in relation to fever), conjuctival injection, facial flushing, coated tongue with pink edges and leukopenia. Abrupt resolution of fever and other signs may indicate the period of remission. Signs of intoxication include icterus, hemorrhage (e.g., epistaxis, hematemesis, melena, metrorrhagia), albuminuria (day 3 or 4), azotemia, shock and encephalopathy. Encephalopathy is not uncommon, with patients usually dying within 7-10 days of onset.

A number of laboratory tests can be supportive in making the diagnosis of yellow fever (Table 2). Serologic testing can definitively diagnose yellow fever in a single sample by early testing for immunoglobulin $M$ by an enzyme-linked immunosorbent assay (ELISA) or in paired acute

Table 1: Questions to ask when determining if a patient has yellow fever ${ }^{3}$

\begin{tabular}{|c|c|}
\hline Question & Rationale \\
\hline Where have you travelled recently? & $\begin{array}{l}\text { Yellow fever is endemic in jungle } \\
\text { areas of Africa and South America }\end{array}$ \\
\hline When did you travel there? & Incubation period is generally $3-6 \mathrm{~d}$ \\
\hline $\begin{array}{l}\text { Which immunizations have you received and } \\
\text { when were they last administered? }\end{array}$ & $\begin{array}{l}\text { Yellow fever can be prevented } \\
\text { through immunization every } 10 \mathrm{yr}\end{array}$ \\
\hline
\end{tabular}

Table 2: Investigative tests and predicted results with yellow fever

\begin{tabular}{ll}
\hline Test & \multicolumn{1}{c}{ Result } \\
\hline Complete blood count & Leukopenia and thrombocytopenia \\
Bilirubin, serum aspartate & \\
aminotransferase, alanine & Elevated \\
aminotransferase & Usually normal \\
Alkaline phosphatase & Elevated \\
BUN and creatinine & Albuminuria (seen by day 4) \\
Urinalysis & IgM or acute and convalescent IgG using ELISA \\
Serology &
\end{tabular}

Note: $\mathrm{BUN}=$ blood urea nitrogen, $\lg \mathrm{M}=$ immunoglobulin $\mathrm{M}, \lg \mathrm{G}=$ immunoglobulin $\mathrm{G}, \mathrm{ELISA}=$ enzyme linked immunosorbent assay. 
and convalescent sera samples. The ELISA method is preferred because of its sensitivity and relative specificity, but neutralization tests provide the highest specificity.

Liver biopsies show a characteristic but not diagnostic midzone necrosis with sparing of hepatocytes around the central vein and portal triad. Viral antigen has been found in degenerating hepatocytes and Councilman bodies.

Treatment is supportive; it should involve intensive care in severe cases and consultation with infectious disease specialists.

Prevention: Yellow fever is readily prevented by vaccinating residents of and travellers to endemic zones. The yellow fever vaccine is a live attenuated strain of the yellow fever virus (17D) developed by Theiler and Smith in 1927. The WHO currently approves only 5 manufacturers of the yellow fever vaccine. Countries located in yellow fever endemic areas may officially require proof of yellow fever vaccination as a condition of entry under WHO international health regulations. There are also countries outside of the endemic areas that may require proof of vaccination because such yellow fever-free countries have the appropriate climatic and entomologic conditions to initiate and maintain a yellow fever transmission cycle. Trav- ellers are asked to consult travel medicine specialists for specific requirements.

The vaccine is approved for children older than 9 months, but because it is an attenuated live vaccine it is contraindicated in pregnancy though safe for breastfeeding mothers. It is generally contraindicated in immunocompromised people, though individual risk assessments are necessary for such cases. Waivers approved by the WHO can be provided to travellers with contraindications for the vaccine by certified travel medicine centres. Such patients should be counselled on prevention methods. The yellow fever vaccine has a long record of safety, but clinicians should be aware of 2 severe complications from the vaccine. Yellow feverassociated neurotropic disease (previously known as postvaccine encephalitis), most common in infants, occurs 7-21 days after vaccination. Of the 1/8 000000 people who contract this disease, full recovery is typical. Yellow fever-associated viscerotropic disease occurs 2-5 days after vaccination. It is characterized by fever, myalgia, arthralgia, increased liver enzymes and bilirubin, lymphopenia, thrombocytopenia, disseminated intravascular coagulation, hypotension, oliguria and rhabdomyolysis. There have been 13 cases reported out of over 100 million doses adminis- tered worldwide. ${ }^{4}$ The vaccine should be given no less then 10 days before departure, and revaccination should occur every 10 years. The vaccine should only be administered by certified vaccine centres knowledgeable in travel medicine and vaccine reaction management, particularly if concurrent administration of other vaccines is indicated, because these may need to be staggered. People with an allergy to egg proteins or gelatin should not be given the yellow fever vaccine. ${ }^{4}$

\section{Erica Weir}

Associate Medical Officer of Health

Kingston, Frontenac and Lennox \& Addington Health Unit

Kingston, Ont.

Shariq Haider

Division of Infectious Diseases

McMaster University Medical Centre Hamilton, Ont.

\section{References}

1. Strickland GT. Hunter's tropical medicine and emerging infectious diseases. 8th ed. Philadelphia: WB Saunders Company; 2000.

2. Monath TP, Cetron MS. Prevention of yellow fever in persons traveling to the tropics. Clin Infect Dis 2002;34 (10):1369-78.

3. Ferri FF, Pearson RL, Opal SM, Vyas JM, Alonso PG. Differential diagnosis. St. Louis (MO): Elsevier; 2003 Oct 27. Available: www.firstconsult.com/home /us_disorder/01014617/part3.htm (accessed 2004 May 26).

4. Cetron MS, Marfin AA, Julian KG, Gubler DJ, Sharp DJ, Barwick RS, et al. Yellow fever vaccine. Recommendations of the Advisory Committee on Immunization Practices (ACIP), 2002. MMWR Recomm Rep 2002;51 (RR-17):1-11; quiz CE1-4.

\section{Holiday Review 2004 Call for Papers}

We're looking for creative contributions for CMAJ's Holiday Review 2004. Humour, personal reflections, history of medicine, off-beat scientific papers and postcards from the edge of medicine are welcome.

Send your offering to the Managing Editor, Josephine Sciortino (800 663-7336 x2366; josephine.sciortino@cma.ca). Articles should be no longer than 1200 words, and photographs or illustrations are encouraged.

The deadline for submissions is September 13, 2004.

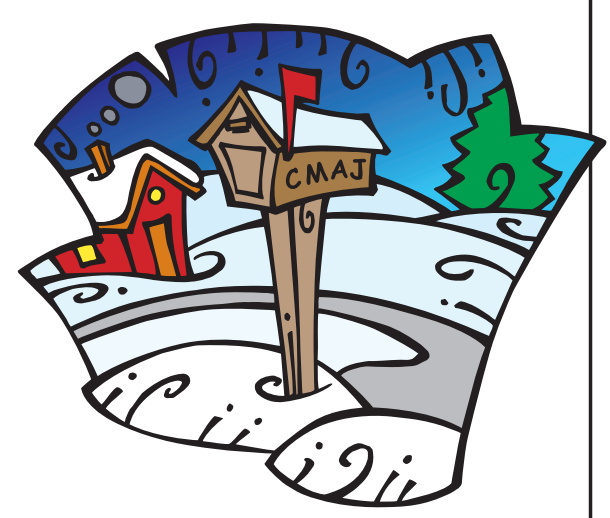

